\title{
Retrospective Medical Record Research: Reflections of A Unsponsored Researcher
}

\author{
Fidelia Bature $^{1,4 *}$, Jitka Vsectekova ${ }^{2}$, Dong Pang ${ }^{3}$, Yannis Pappas ${ }^{3}$ and Barbara Guinn ${ }^{4}$ \\ ${ }^{1}$ Faculty of Health and Social Studies, School of Applied Social Studies, University of Bedfordshire, UK \\ ${ }^{2}$ Faculty of Wellbeing, Education \& Language Studies, School of Health, Wellbeing and Social Care, UK \\ ${ }^{3}$ Institute for Health Research, Putteridge Bury Campus, University of Bedfordshire, UK \\ ${ }^{4}$ Department of Biomedical Sciences, Faculty of Health Sciences, University of Hull, UK
}

*Corresponding author: Fidelia Bature, Faculty of Health and Social Studies, School of Applied Social Studies, University of

Bedfordshire, Luton, LU1 3JU, UK

ARTICLE INFO

Received: 幽 September 24, 2019

Published: 絊October 01, 2019

Citation: Fidelia B, Jitka V, Dong P, Yannis P, Barbara G. Retrospective Medical Record Research: Reflections of A Unsponsored Researcher. Biomed J Sci \& Tech Res 21(4)-2019. BJSTR. MS.ID.003642.

Abbreviations: AD: Alzheimer's Disease; CCG: Clinical Commissioning Group; CPRD: Clinical Practice Research Database; CRN: Clinical Research Network; GP: general practitioner; MK: Milton Keynes; NIHR: National Institute for Health Research; REC: Research Ethics Committee; RMRR: Retrospective medical record review; STEM: Science, Technology, Engineer and Mathematics

\begin{abstract}
This article is a reflective narrative by the author following the successful completion of a doctoral thesis involving the analysis of primary care data. The aim of the study was to identify patterns in signs and symptoms preceding a clinical diagnosis of Alzheimer's Disease (AD). Here, we discuss the challenges of conducting research using the patient medical health records as a non-sponsored researcher. The narrative explores the experiences of undertaking practice recruitment, issues that arose around access to data and the requirement for stakeholder commitment, with proposals for other similar projects that attempt to undertake research using patients' data. The Gibbs reflective cycle was adopted in this narrative. This model encourages a clear description of events, analysis of personal feelings, evaluation of the experience, conclusions as to the options that were considered and actions to take if faced with the same study methods (involving primary care practitioners and/or the review of patient records). It also allows a structured reflection while simultaneously discussing the barriers and facilitators to undertaking a retrospective medical record research. The researcher also proposes some recommendation(s) to overcoming barriers to recruitment in primary care.
\end{abstract}

Keywords: Retrospective Medical Record Review; Unsponsored Researcher; General Practice; Interview; Reflection
Aims
To review the difficulties faced when accessing patient medical records from primary care
To recommend best practice that can help circumvent some of the difficulties faced by a non-sponsored researcher

\section{Introduction}

Retrospective Medical Record Review (RMRR), that collates General Practitioners (GPs) interpretation of patients' personal experiences, can be challenging. This article presents the difficulties faced by an international student while undertaking a doctoral research project that was designed to encompass multiple GP practices and partners to ensure broad applicability. During this time, research was undertaken to identify patterns in the signs and symptoms preceding the clinical diagnosis of Alzheimer's Disease (AD), using a RMRR.

$\mathrm{AD}$ is a condition that affects the cognitive and functional abilities of individuals. The disease is associated with stigmatisation $[1,2]$ due to a lack of insight by patients and understanding by their carers, the anticipation of the course of the disease, and general public perceptions of $\mathrm{AD}[1,3]$. The fear of a loss of independence 
and particularly of becoming redundant can affect patients psychologically and appears to be the main reason that the reporting of memory loss does not happen in a timely manner [1].

While all research presents consequences of some kind, RMRR is increasingly being used to identify issues regarding health and disease [4] but is sensitive in nature. This is because it comes with (potential) ramifications especially when dealing with issues regarding ethics and stigma, as presented in most diseases but especially those involving cognitive decline such as AD [1]. Medical records present the opportunity to undertake studies without the time-consuming process of recruiting individuals for longitudinal studies; it can reduce the financial burden of a real-time study. However, they are limited in their content, influenced by GP record taking and present ethics committees with concerns around the invasion of privacy, the security of information and the integrity of the representation of patients' data $[5,6]$.

There is a risk that the review of medical records could identify other potentially sensitive health conditions [7] and patients are often not consented in anticipation of a researchers' review of their medical records. The RMRR study was undertaken to meet the requirement of a longitudinal study in this research while recognizing the challenges associated with it. Several ethical and methodological issues were observed during this study including the difficulties of recruiting GPs because accessibility to them often comes via practice managers who often act as primary care gatekeepers. Other challenges included time constraints on GPs, a lack of resources and on occasion, a lack of cooperation by practice managers that appeared to conflict with the GPs own willingness to participate. There were also issues of financial incentives. These factors impact negatively on all research. Despite unlimited enthusiasm by the researcher and a willingness to continue to pursue leads of recruiting GP practices in two regions, very few of those who initially seemed keen to help, ultimately engaged with the research. Discussing such challenges openly will enable a wider discussion around methodological approaches to research such as RMRR and the possibility of undertaking sensitive research differently in the future. For example, the Clinical Practice Research Database (CPRD) presents an opportunity to explore patient's data to enable change without the challenging process of recruiting GPs and their practices.

\section{Background}

Ethical approval was obtained from the NHS-Research Ethics Committee (REC) of North of Scotland and London, who provided opportunities for the student researcher to engage in individual research projects within the NHS. The researcher began the process of recruiting GP practices in 2015 with personal visits and emails to fulfil the requirement for the Ethics Board; these were followed with another email in 2016 after the meeting and approval of the study by the CCG contact. The research phase was completed in 2017, with seven GPs in the interview phase and five practices for the RMRR study. The underlying reasons for the limited participation as stated by the practices included limited human resources, managerial and gatekeeper issues, as well as the lack of financial compensation/ incentives that the researcher could offer to practices.

Recruitment of practices could be challenging and slowed down the progress of research to the extent that it could ultimately lead to the termination of the study; however, in this research, it did slow down the progress and extended the time of completion. The issues faced could be overcome by extending the original proposed time; reducing the power of generalization and the inflation of the overall cost of the research, each of which could present an enormous challenge to a unsponsored researcher [8] and could have ethical implications in and of itself especially when there is no justification for refusal. It has been estimated that less than one-third of primary care studies successfully recruit health care practices within the originally proposed time-frame in the UK [9]; the success becomes less with an unsponsored researcher as indicated in this research. Even when the research team is made of a number of researchers, there is difficulty in practice recruitment [10], which may hinder ideas being translated to solutions, while the need to base actions on researched evidence continues.

Being a self-funded $\mathrm{PhD}$ student, and in some senses a lone researcher, comes with its rewards. It is a time of great interest and sober reflection and offers the opportunity to climb to great heights unhindered by considerations of a team of data collectors. There is also the tendency for long and detailed investigations in order to be precise and not to miss any opportunity, made much harder by working alone because of the time taken to collect the same amount of data. The isolation can make it easier for the lone researcher to quit the game entirely, especially where slow progress prevents the collection of enough data for analysis within time constraints. The early part of the PhD researchers' career is also a point recognised as the creative and energetic part of the researcher's research journey [11]. This can be enhanced with adequate support from the PhD researchers' supervisors as well as the wider community where the researcher hopes to engage.

However, little attention has been given to self-funding, often time international researchers, who regularly work additional uncompensated hours to translate their growing research ideas into opportunities worthy of external support $[12,13]$; this means that important contributory opportunities are missed or lie dormant due to the non-capitalisation of the expertise of the unsponsored $\mathrm{PhD}$ researcher

\section{Narrative Approach and Reflection of Events}

Till date, there has been no publication on how the recruitment process in the NHS influences a $\mathrm{PhD}$ student or self-funded researchers' view of their data collection. Besides highlighting the recruitment process in the NHS and the impact of researching within the primary care system upon an often-isolated researcher, this study presents a constructive view of the impact of the recruitment process on the field researcher. After much effort 
in terms of time and finances, there were several recruitment challenges that suggested of the predictive model impossible; these challenges will be discussed in turn. It has been suggested that the research topic, invitation method, practice/GPs interest in the research and managerial issues could present challenges in the recruitment process [14].

Earlier, Solberg et al. [15] identified seven research factors as determinants for a successful recruitment in health care professionals including relationships with the local community; reputation of the researcher with the host community; requirement or resource demand; rewards in kind, cash and recognition; reciprocal as in negotiation; resolution in time or recruitment set time, and respect for participants duties and constraints as not to be taken for granted [10]. Consequently, the researcher and her supervisors came up with a topic that was interesting to physicians and patients alike, based on our own interests in AD, and the belief that RMRR offered a unique way to screen practice notes retrospectively for common signs preceding the development of a clinically diagnosed disease (in many cases by decades rather than weeks or years). Different methods of recruitment were employed including an interesting topic, adequate contact with gatekeepers, personal visits, emails, telephone calls, and pair recruitment, etc.

It was then set out to invite the general practices in Milton Keynes (MK), the researcher's hometown to participate in the study. Not only did the researcher visit the GPs practices as part of the recruitment, but one of the supervisors accompanied the researcher to meet with the Clinical Commissioning Group (CCG) contact as a gatekeeper to increase the chances of GP recruitment. The CCG contact responded positively and immediately drafted an email to all GPs and their practices in the MK area. However, this only achieved a response from one GP practice manager, who responded but later declined for reasons that will be explained below. The response rate for the recruitment was very low with only seven practices agreeing to enter a discussion following a personal invitation by the researcher and pair recruitment initiated by the supervisor. There was variability with regards to who in the practices responded to our invitation (e.g. nurse, practice manager, GP).

The initial written invitation sent through email from the researcher was followed up by phone calls and personal visits to the practices to facilitate practice participation. Larger sample sizes normally improve the ability of researchers to generalise about their findings [16] and we strived for this in our research. Throughout this process, we noted five matters that affected participation and sampling in this study as follows:

a) General practices operate within a culture of financial incentives, which has become the norm for their participation in studies. This is a known fact from several other programmes of research [17-20]. The manager, that the researcher met in person, showed interest and gave another date for a more detailed meeting in order to have an opportunity to discuss the study with the GP partners in the practice. The researcher came back full of hope but at this meeting, the manager requested for financial recompense for her and the GPs; she mentioned that this was standard practice before they could participate in any research. The researcher explained to her that this was a $\mathrm{PhD}$ project without sponsorship but with ethical approval from the university and the NHS REC. However, the manager insisted that payment was a condition of participation and then affirmed that a meeting will be set again with the partners and communicate with the researcher within two weeks.

Two weeks came and passed without contact and the researcher proceeded to give her a call the following week without success. The subsequent three visits yielded no outcome as on each occasion; the researcher was told that the manager was so busy that she could not meet or speak with the researcher on the phone.

b) Practices have no clear guidelines as to how and by whom a study will be assessed before they decide to engage. If such guidelines existed, in our experience, they weren't shared. The ethics approval is based on these practices indicating their interest, however, how this is sought, is unclear. The only manager, who responded to the CCG contact email, emailed the researcher and fixed an appointment to meet in the practice for the interview and data collection. Unfortunately, when the manager learned that she wasn't going to be interviewed, she declined to participate on the grounds that she thought she would be interviewed not the GPs and subsequently insisted that her GPs were very busy and regret that their non-participation.

c) There was a lack of time and personnel to anonymise the data before it was seen by the researcher. It was always "I am sorry, this is our busiest time and we cannot participate". When the researcher requested to give some time and to come back, the response was "I am sorry, we would have to say no at this time." Some GP practices were visited up to four times and the receptionist would suggest the lone student to come back again and again. In six independent practices, the practice managers emphasised that their GPs will not participate based on the current crisis in primary care including case overload and shortage of staff (Iliffe, 2018).

d) Requests for access by the researcher were sometimes dealt with as if personal favour was being asked. The researcher was fortunate to meet with six managers personally and on three occasions, the manager would greet a Caucasian female and state "Hi, you must be the researcher!" to which the black researcher rose and responded in the affirmative. One of the managers kept stating that they were busy and asking the researcher to return at another time. On the fifth visit, the manager greeted the researcher by shouting; "We don't have any vacancy here"; the researcher then insisted that she was not there to look for a vacancy but to present the document that was requested at the last discussion. 
Even though the manager apologised and said "I thought that you the black lady that had been here earlier to look for a job"; she further postponed the meeting, requested that the researcher visit other GP practices that have signed up for the study and find out the system that they were using to anonymise their data. Luckily, one of the practices obliged this information, which again was presented to the manager, who at this time, bluntly stated that the practice could not participate. This is an indication that individual gatekeepers influenced the positioning of the researcher.

e) There are physicians who take time to support studies that are close to their interests without requesting a reward. Although such initiatives are commendable and do enable studies to be conducted, the very small number physicians who might decide to support a study because of their interests, were insufficient in numbers to provide our studies with the generalisability it could have generated. Also, regardless of how important such initiatives may be to individual studies, there is a lack of understanding of how a decision is made to support or not to support a study by a practice. One of the GPs was enthusiastic about participating but would only do so when his practice manager consented to his participation; however, the practice manager refused to consent on the grounds that they had too many patients with too few GPs. This supports Loskutova et al. [14], who reiterated that most recruitment challenges represent managerial challenges, who are sometimes not experienced enough to decide on the importance of a study.

\section{Discussion}

In this reflective piece, we have mentioned five challenges that impacted negatively on a research project that had the potential to contribute positively to patients' outcome. We elaborate as follows: Government funding and subsidisation provide general practices with operational sustainability, as their role in the community is recognised as paramount. The culture of financial support has been extended to provide individual practices with financial rewards for participating in public health programmes such as those of integrated care and research $[19,20]$. GPs are normally financially rewarded for their participation in local and national committees [18]. Within this culture of financial compensation for participating in public health programmes and research, programmes of research which do not have access to funds to provide financial rewards to general practices may never materialize or may not produce the maximum impact needed especially with the emphasis on evidence-based practice.

Indeed, the request by practices for compensation for the anonymization of patient notes prior to release to a researcher is not an unreasonable one, especially if the monies provided by government funding are entirely accounted for. However, most researchers without sponsors do not have the capacity to provide financial compensation/incentives for their participation and there are controversies as to the extent to which these incentives are responsible for the improvement in quality care and their benefit to patients' outcomes $[18,20]$. Whilst we recognise the importance of financial viability to practices, there need to be efforts to facilitate good research that enriches health care in the UK and positively impacts local communities including our understanding of disease initiation, and progression, for those that care for others.

Funding by outside organisations such as the National Institute for Health Research (NIHR) Clinical Research Network (CRN) are highly competitive, provide focussed research support and are timeconsuming to apply for. Moreover, funding from these organizations is not always available for international self-sponsored students. The lone researcher is often underfunded, in contrast to large wellfunded groups, and spends their own time, and money, gathering research data. Guidance setting out the pathways in which GPs can support research, that benefit practices that engage with some studies, is needed. Clear guidelines/financial support for research will not only facilitate the non-sponsored and lone researcher but provide a greater contribution to STEM and healthcare knowledge for the evidence-based practice advocated by the U.K. government.

While Section 3.7 of the Health Research Authority (HRA) Ethics Guidance to Payment and Incentives in Research states that "Where the risk and burdens of the research are considered by a REC to be justified by the potential benefits then it will normally be acceptable for competent adults to participate in the research study without being paid ", Section 3.8 indicates that "Where it is considered ethically acceptable for individuals to take part in a study for no payment it would also be acceptable to pay individuals for participation in that study proportionate to the level of burdens involved and/or (justified) risk". However, payment in proportion to the level of involvement like data collection should clearly be defined and generalized for researchers to have an anticipation of what they are involved in.

Gatekeeping plays an important role in facilitating research access, as it determines whether research poses a threat to the participants or the public at large or offers benefits to society. It is understood that vigilance by gatekeepers is important especially when this has to do with patients' data, which requires careful consideration of the ethical implications and the approvals given [21]. However, it is difficult, as in our case, to determine the motives of the gatekeepers and their degree of influence on GPs; or whether they are in fact just following instructions from the GPs, who are indeed, their line managers. Furthermore, RMRR gatekeepers should consider and emphasise the issues of access and possibly limit their roles in preventing research from progressing, especially when ethics has been approved by the issuing authority. Permission should be given solely in consideration of the resource implications and the expected time contribution required by the research.

Ironically, research requires continuous negotiation with gatekeepers, which is a demonstration of transparency and respect for institutional autonomy $[21,22]$, but how long these negotiations 
should take and what is required to make it through these practice barriers is not clear. Should research be delayed just to wait for the gatekeeper's permission? Careful consideration and respect for the time of the researcher are also needed. Indeed although some GPs were incredibly helpful and supportive of the research, the unnecessary time and cost associated with referring the researcher to a gatekeeper whose job it was to say 'maybe', 'come back' and eventually 'no', could have been circumvented by a more direct and honest answer at the first contact, saving everyone involved time and frustration. Had gatekeepers/GPs given a clear 'no' at the first meeting, the researcher would have had more time to pursue new leads in a wider area, instead of chasing potential leads in a diminishing time-frame.

This was particularly frustrating when the researcher was asked to return to the practice over and over again, in some instances up to six times. This said and done, it could indicate that there was a willingness, however, without a means in time or resources. Power dynamics also play a part in how research is undertaken within the health and social care. This also had a part in our research as indicated in the narratives. The reason has been attributed to weak and opaque management; inadequate guidelines for research and development facilitated by primary care providers, failure to recognize the potential of engaging in research and the support that research contributions need, as well as the lack of clear guidelines and definition of roles [23]. Ironically, we have witnessed some management that have engaged in research because of the contribution that the research will make on healthcare development in general, often based on their own areas of interest.

This is a call to educate gatekeepers on the great contribution that research can bring to our understanding of disease but most importantly, how they, or indeed GPs communicate with researchers. Once a proposal is peer-reviewed and approved, general guidance to encourage or indeed force government-funded GP practices to engage with some hours per month or year with research could facilitate research-led improvements in primary care. The provision of a letter from the GP to the practice manager agreeing to engage and a quick tick box form for the practice manager to return to the research explaining if the practice won't engage why not (Figure 1) may have circumvented a number of face-to-face meetings between the researcher and practice that ultimately led to no progress in the research. Even though we are advocating for researchers to make use of the databases created for patients and accept that these databases can help avoid the challenges we faced, electronic health records are not suitable for many research questions, especially without the explicit knowledge of the complexities and limitations of these types of data [6]. These complexities arise out of the variation in health care processes across countries, as well as issues of safety and security around patient data [5,24]. Furthermore, to access the data requires finances, which might not be available to the lone or self-financed researcher.
(A)

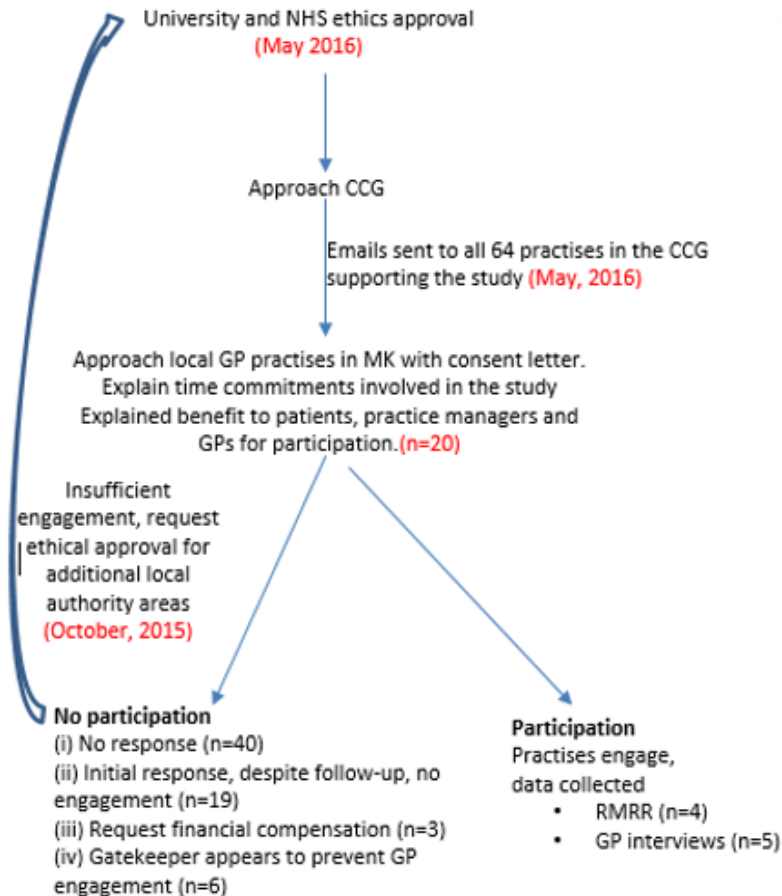

(B)

University and NHS ethics approval for primary care practises (anticipate an approximate $5 \%$ engagement over a 2 years period available for RMRR data collection)

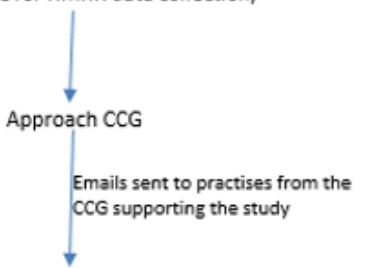

- Approach local GP practises, in person and with letter to named GP

Explain financial compensation is not possible (or offer financial compensation if available).

- Explain time commitments involved in the study.

Explain benefits to patients, practise managers and GPs of participation

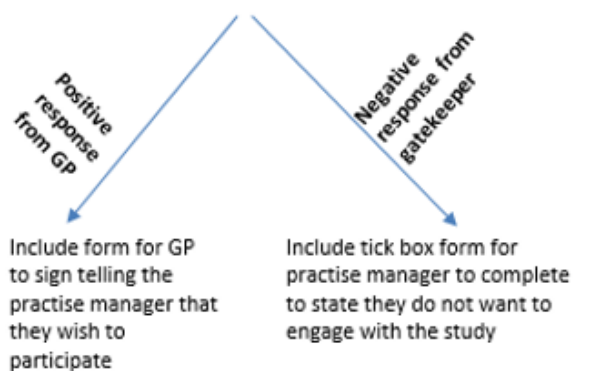

Figure 1: Process of recruitment (A) process followed (with numbers involved $(n=)$ or date completed in parentheses); (B) following experience and reflection - process recommended to future investigators. 
Patients' medical records present an opportunity for researchers to deliver more efficient studies in our case by identifying patterns in signs and symptoms of diseases, which can help in the early diagnosis of diseases and deliver timely healthcare intervention. However, with General Practices in crisis [25], the workload has become more complex and intensive without the funds to support the workforce and the complex infrastructure required [26]. As the pressure has grown, patient experience has also deteriorated [27-36], with a knock-on effect that the recruitment of practices for research that would contribute in improving patients'experience has become almost impossible. We advocate for more funding and enlightenment in terms of what research means to healthcare development, on how patient notes can be made accessible for those with appropriate ethical approvals in place.

This would set a high standard on how non-sponsored and/or lone researchers can be encouraged to continue to contribute to research in the NHS, often at a personal financial cost. Additionally, it would also diminish the number of researchers approaching GPs and PC practices for other than when it is essential to do so that these studies could be better engaged with. Acknowledgements: The Luton, Dunstable and Milton Keynes General Practise consortium helped with the data for this publication and includes Drs Arnold Berger, Mokshad Kansagra and Kulsoom Ali of Fishermead Medical Centre, Buckinghamshire, MK6 2ER; Dr Manoj Sekharan of Milton Keynes Village Practice, Buckinghamshire, MK10 9BQ; Dr Christiane Woloniecki of Woodland Avenue Practice, Bedfordshire, LU3 1RW ; Dr Emma Chakravarty of Leagrave Surgery, Bedfordshire, LU4 9QZ and Dr Anthea Robinson, Moakes Medical Centre, Bedfordshire, LU3 3SR.

\section{References}

1. Abojabel H, Werner P (2019) Exploring family stigma among caregivers of persons with Alzheimer's disease: The experiences of Israeli-Arab caregivers. Dementia 18(1): 391-408.

2. Agniel D, Kohane IS, Weber GM (2018) Biases in electronic health record data due to processes within the healthcare system: a retrospective observational study. BMJ 361: 1479.

3. Baird B, Charles A, Honeyman M, Maguire D, Das P (2016) Understanding pressures in general practice. London: King's Fund pp. 100.

4. Brodaty H, Gibson LH, Waine ML, Shell AM, Lilian R, et al. (2013) Research in general practice: a survey of incentives and disincentives for research participation. Mental health in family medicine 10(3): 163.

5. Button KS, Ioannidis JP, Mokrysz C, Nosek BA, Flint J, et al. (2013) Power failure: why small sample size undermines the reliability of neuroscience. Nature Reviews Neuroscience 14(5): 365-376.

6. Confait MF (2018) Maximising the contributions of PHD graduates to national development: the case of the Seychelles.

7. Cornelius VR, McDermott L, Forster AS, Ashworth M, Wright AJ, et al. (2018) Automated recruitment and randomisation for an efficient randomised controlled trial in primary care. Trials 19(1): 341.

8. Doran T, Kontopantelis E, Valderas JM, Campbell S, Roland M, et al. (2011) Effect of financial incentives on incentivised and non-incentivised clinical activities: longitudinal analysis of data from the UK Quality and Outcomes Framework. Bmj 342: 3590.
9. Draper H, Wilson S, Flanagan S, Ives J (2009) Offering payments, reimbursement and incentives to patients and family doctors to encourage participation in research. Family practice 26(3): 231-238.

10. Dubois B, Padovani A, Scheltens P, Rossi A, Dell'Agnello G (2016) Timely diagnosis for Alzheimer's disease: a literature review on benefits and challenges. Journal of Alzheimer's disease 49(3): 617-631.

11. Ewing G, Rogers M, Barclay S, McCabe J, Martin A et al. (2004) Recruiting patients into a primary care-based study of palliative care: why is it so difficult? Palliative Medicine 18(5): 452-459.

12. (2019) Health Research Authority Ethics Guidance. Payment and Incentives in Research.

13. Høyland S, Hollund JG, Olsen OE (2015) Gaining access to a research site and participants in medical and nursing research: A synthesis of accounts. Medical Education 49(2): 224-232.

14. Iliffe S (2018) Healthcare on the brink? Assessing the crisis in General Practice. Renewal 26(2): 76-88.

15. Kars MC, van Thiel GJ, van der Graaf R, Moors, M, de Graeff, et al. (2016) A systematic review of reasons for gatekeeping in palliative care research. Palliative medicine 30(6): 533-548.

16. Loskutova NY, Smail C, Ajayi K, Pace WD, Fox CH (2017) Recruiting primary care practices for practice-based research: a case study of a group-randomized study (TRANSLATE CKD) recruitment process. Family practice 35(1): 111-116.

17. McDonald AM, Knight RC, Campbell MK, Entwistle VA, Grant AM, et al. (2006) What influences recruitment to randomised controlled trials? A review of trials funded by two UK funding agencies. Trials 7(1): 9.

18. McRae AD, Perry JJ, Brehaut J, Brown E, Curran J, et al. (2018) Engaging emergency clinicians in emergency department clinical research. Canadian Journal of Emergency Medicine 20(3): 443-447.

19. Minchin M, Roland M, Richardson J, Rowark, S, Guthrie B (2018) Quality of care in the United Kingdom after removal of financial incentives. New England Journal of Medicine 379(10): 948-957.

20. Morténius H, Biagi A, Palm L, Fridlund B, Björkelund C et al. (2015) Impact of the organisational culture on primary care staff members' intention to engage in research and development. Journal of health organization and management 29(2): 234-251.

21. Nicholas D, Watkinson A, Boukacem Zeghmouri C, Rodríguez Bravo B, $\mathrm{Xu}$ J, et al. (2017) Early career researchers: scholarly behaviour and the prospect of change. Learned Publishing 30(2): 57-166.

22. Parkinson A, Jorm L, Douglas KA, Gee A, Sargent GM, et al. (2015) Recruiting general practitioners for surveys: reflections on the difficulties and some lessons learned. Australian Journal of Primary Health 21(2): 254-258.

23. Pollock AM, Roderick P (2018) Why we should be concerned about accountable care organisations in England's NHS. BMJ 360: 343.

24. Price M, Bellwood P, Kitson N, Davies I, Weber J, et al. (2015) Conditions potentially sensitive to a personal health record (PHR) intervention, a systematic review. BMC medical informatics and decision making 15(1): 32.

25. Rathert C, Porter TH, Mittler JN, Fleig Palmer M (2019) Seven years after Meaningful Use: Physicians' and nurses' experiences with electronic health records. Health care management review 44(1): 30-40.

26. Riis A, Jensen CE, Mandal HT, Bro F, Jensen MB (2016) Recruitment of general practices: Is a standardised approach helpful in the involvement of healthcare professionals in research?SAGE open medicine 4, p.2050312116662802.

27. Sarkar S, Seshadri D (2014) Conducting record review studies in clinical practice. Journal of clinical and diagnostic research: JCDR 8(9): JG01JG04. 
28. Schreijenberg M, Luijsterburg PAJ, Van Trier YDM, Rizopoulos D, Koopmanschap MA, et al. (2018) Discontinuation of the PACE Plus trial: problems in patient recruitment in general practice. BMC musculoskeletal disorders 19(1): 146.

29. Shaw A, De Lusignan S, Rowlands G (2005) Do primary care professionals work as a team: a qualitative study? Journal of interprofessional care 19(4): 396-405.

30. Singh S, Wassenaar DR (2016) Contextualising the role of the gatekeeper in social science research. South African Journal of Bioethics and Law 9(1): 42-46.

31. Sizmur S, Raleigh V (2018) The risks to care quality and staff wellbeing of an NHS system under pressure. The King's Fund: Oxford p. 24.

32. Slade SC, Philip K, Morris ME (2018) Frameworks for embedding a research culture in allied health practice: a rapid review. Health research policy and systems 16(1): 29.

\section{ISSN: 2574-1241}

DOI: 10.26717/BJSTR.2019.21.003642

Fidelia Bature. Biomed J Sci \& Tech Res

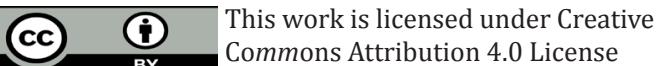

Submission Link: https://biomedres.us/submit-manuscript.php
33. Solberg LI (2006) Recruiting medical groups for research: relationships, reputation, requirements, rewards, reciprocity, resolution and respect. Implement Sci 1: 25.

34. Stites SD, Johnson R, Harkins K, Sankar P, Xie D, et al. (2018) Identifiable characteristics and potentially malleable beliefs predict stigmatizing attributions toward persons with Alzheimer's disease dementia: Results of a survey of the US general public. Health communication 33(3): 264273.

35. Weissman JS, Saglam D, Campbell EG, Causino N, Blumenthal D (1999) Market forces and unsponsored research in academic health centers. JAMA 281(12): 1093-1098.

36. Yüksel B, Küpçü A, Özkasap Ö (2017) Research issues for privacy and security of electronic health services. Future Generation Computer Systems 68: 1-13.

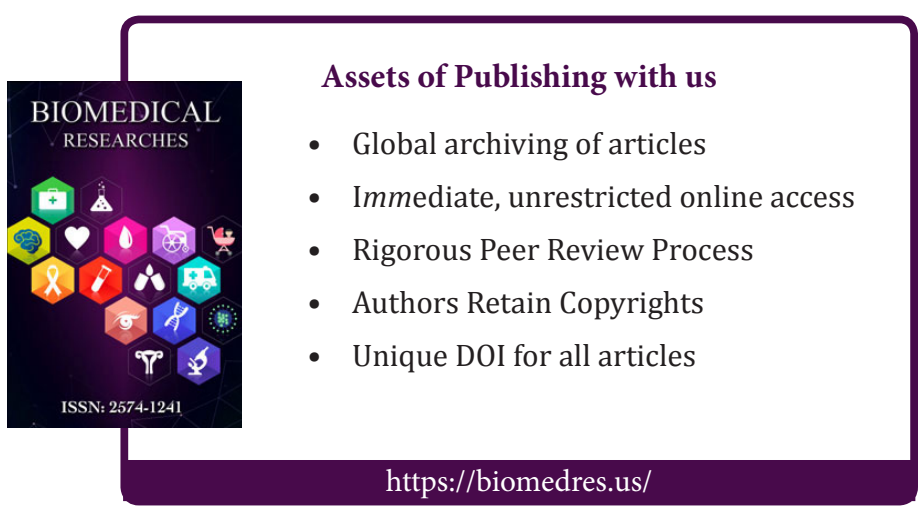

